\title{
Maximizing the community exploitation of the VLTI 2nd-generation instruments
}

\author{
Stefan Kraus $^{1}$ (D) Paulo Garcia ${ }^{2} \cdot$ Guy Perrin $^{3}$
}

Received: 30 November 2017 / Accepted: 2 March 2018 /Published online: 3 April 2018

(C) The Author(s) 2018

Keywords Very Large Telescope Interferometer:VLTI · Optical interferometry · Infrared interferometry · Community exploitation of VLTI · GRAVITY · MATISSE · PIONIER

The Very Large Telescope Interferometer (VLTI, Fig. 1) is the European flagship interferometric facility and allows European astronomers to study the universe on milli-arcsecond scale, enabling fundamentally new opportunities in planet formation to stellar and extragalactic astronomy. The facility was conceived with the goal of making optical interferometry available to the whole European astronomy community and to serve the needs both of expert as well as non-expert users. The VINCI commissioning instrument [5], the 1st'generation instruments MIDI [7] and AMBER [9] and the visitor instrument PIONIER [6] took major steps towards this goal but revealed also challenges, for instance, related to attracting non-expert users to interferometry. Currently, the VLTI undergoes a major transformation with the arrival of the 2nd-generation instruments GRAVITY [4] and MATISSE [8]. GRAVITY has been offered to the community since October 2016, while the on-sky commissioning of MATISSE is due to start in early 2018, with a possible start of regular science observations the following year. In this contribution, we reflect on how the 2ndgeneration instruments might help in expanding the VLTI user community and we will discuss steps that could be taken to support this process. The expert community, both inside and outside of the instrument consortia, should coordinate in order to optimize the scientific output of the new VLTI instruments.

Stefan Kraus

1 Exeter, UK

2 Porto, Portugal

3 Paris, France 


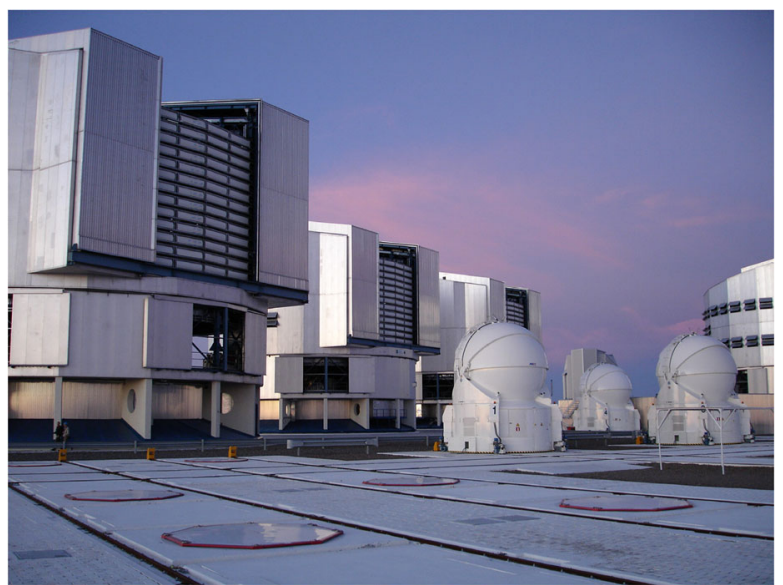

Fig. 1 The Very Large Telescope observatory features four $8.2 \mathrm{~m}$ unit telescopes, four $1.8 \mathrm{~m}$ auxiliary telescopes, and the infrastructure for performing optical interferometry. Image Credit: S. Kraus

Potential for extending the user base of VLTI Being 4-telescope beam combiners, both GRAVITY and MATISSE offer a distinct advantage for attracting new VLTI users, as they will be much more efficient in data collection than the 2- and 3-telescope beam combiners MIDI and AMBER. The increased number of observables will also enable a more efficient application of closure phase image reconstruction techniques and allow users to retrieve model-independent images. An important role of these images is to offer guidance with selecting appropriate model geometries for the quantitative model fitting process. ESO and the GRAVITY and MATISSE consortia have realized the importance that imaging might play and will include image reconstruction algorithms in their data reduction package. The existing 4-telescope beam combination instruments CHARA/MIRC and VLTI/PIONIER have shown that meaningful images can be reconstructed if a reasonable amount of observing time is invested (e.g. several nights on different VLTI configurations with the auxiliary telescopes). However, it is important not to raise overly-optimistic expectations, as imaging will continue to be applicable only to retrieve structures in a specific angular size range (e.g. 3-60 mas), contrast range (e.g. <1:50), and of modest complexity. The users of GRAVITY and MATISSE will need to take these aspects into account during proposal planning and will need to gain experience in applying the available image reconstruction algorithms and learn to identify artifacts introduced by the incomplete uv-coverage or by the image reconstruction method. Therefore, the community needs to offer adequate assistance to new GRAVITY and MATISSE users. For this purpose, we are currently in the process of establishing a network of VLTI Expertise Centres that will aid on aspects ranging from observational project planning, data reduction, to image reconstruction and model fitting. These Expertise Centres will organize regular proposal preparation workshops, provide tutorials and software tools on a centralized website, and offer personal assistance to new users. The details are outlined in an article in the whitebook on the Future of Interferometry in Europe (http://www.european-interferometry.eu/workinggroups/the-future-of-interferometry-in-europe) and on the Expertise Centre network website (http://www.european-interferometry.eu/vlti-expertise-centres-network). Another important building block in the strategy to make interferometry more 
accessible to non-experts is provided by the OPTICON-funded working group on image reconstruction. This working group develops a software tool that will allow users to run different image reconstruction algorithms under one graphical interface and to compare the resulting images quantitatively and to investigate how the final product depends on image reconstruction parameters such as the regularization weight (results will be made available on the following website: http://www.european-interferometry. eu/joint-research/fp7-ii-2013-2016-activities).

Importance of promoting science opportunities of VLTI Another important component for expanding the VLTI user base is to promote the scientific opportunities of VLTI more effectively to the non-expert community. Outside of the VLTI user community, many astronomers are not aware of the capabilities provided by optical interferometry nor to its complementarity to other techniques. At present, these capabilities are communicated primarily through individual scientists promoting specific science results on conferences. Continuing and enhancing these individual efforts are very important. At the same time, it is also in the interest of the instrument consortia and of ESO to build a user community for upcoming instruments, for instance by organising dedicated workshops \& community events. Finally, it would be desirable to advertise the capabilities of the VLTI in the context of optical interferometry worldwide, as potential VLTI users might also be interested in making use of the complementary capabilities that are offered by US-based facilities, such as the Center for HighAngular Resolution Astronomy (CHARA) array [10], the Navy Precision Optical Interferometer (NPOI, [1]), and the Magdalena Ridge Observatory Interferometer (MROI, [2]) that is currently under construction. One of these facilities, namely the CHARA array, offers already an open-access route (as part of the NOAO time allocation process) and complements the VLTI with respect to baseline coverage (about 2.5 times longer maximum baseline length: up to $331 \mathrm{~m}$ ), wavelength coverage (down to the visible wavelengths: 0.6 to $2.4 \mu \mathrm{m}$ ), and sky coverage (access to the northern hemisphere: DEC $>-20^{\circ}$ ). It is important to make non-experts aware of these complementary capabilities, which could be facilitated, for instance, by scheduling dedicated talks about the US facilities at European meetings, such as VLTI Community Days. Furthermore, the organisers of the VLTI Community Days and the organisers of the annual CHARA meeting could consider scheduling both meeting at the same location on adjacent days, which would increase the opportunities for both communities to meet, to exchange the latest science results, and to intensify cross-facility collaboration.

Maximizing science output through coordinated/simultaneous observations The expert community should organize itself with the goal of maximizing the scientific return of the VLTI. For instance, it is evident that GRAVITY and MATISSE will, to a large extent, target the same objects, including for instance the brightest active galactic nuclei, many young stellar objects, and some key evolved stars. These observing programs will deliver exciting science in their own right, but in many cases it is clear that coordinated simultaneous objects could yield even more spectacular results. Therefore, users should consider coordinating the execution of MATISSE and GRAVITY observations on time-variable objects at the same epoch, enabling multi-wavelength, time-domain astronomy studies of key objects. This might be particularly relevant for GTO programs and Large Programs that target typically extensive object 
samples and include often also the brightest members of each object class (offering the highest chance that the object might be accessible by several instruments). To support this process, the establishing of an "i-SHOOTER" mode should be considered, where PIONIER, GRAVITY, and MATISSE (Fig. 2) would be able to record data in the H/K/ $\mathrm{L} / \mathrm{M} / \mathrm{N}$-band in parallel. Conducting such multi-instrument observations will require a high degree of coordination and collaboration, but it could offer the teams an opportunity to share the time that is spent and therefore to execute their programs more effectively. There exist already some examples of inter-consortia collaboration, for instance in the ongoing attempt to make the GRAVITY fringe tracking accessible to MATISSE (GRAV4MAT project), and such collaborations could be further extended in order to maximize also the scientific exploitation phase of GRAVITY and MATISSE. At the same time, VLTI users should explore the potential of coordinating simultaneous CHARA, NPOI, or future MROI observations for objects that are accessible from both hemispheres. Depending on the precise science cases, the resulting improvements in uv-coverage or wavelength coverage might greatly enhance the scientific value of the data set, in particular when the observations are conducted nearly-simultaneously for time-variable objects.

Opportunities for scientists outside the GTO consortia Of course, excellent science will also be done outside of the GTO programs. The GTO time provides the instrument teams with a well-earned compensation for their long-term engagement in envisioning, building, and commissioning new instruments for ESO telescopes. Besides the actual guaranteed time, the GTO teams are also awarded the right to protect a list of targets for their programs. In the past, the target protected has been implemented either as a protection over the whole GTO duration (i.e. independent of the specifically planned observations by the GTO team for a given semester; this scheme was adopted for MIDI) or on a semester-by-semester basis (i.e. only those targets are protected which the GTO team actually plans to observe in a particular semester; as adopted for AMBER).

Too stringent target protection policies can form a barrier for non-GTO teams to formulate their own science programs and to the expansion of the VLTI user community. This is particularly evident when the number of accessible targets is already small due to instrument sensitivity limitations, such as in the mid-infrared where only a few
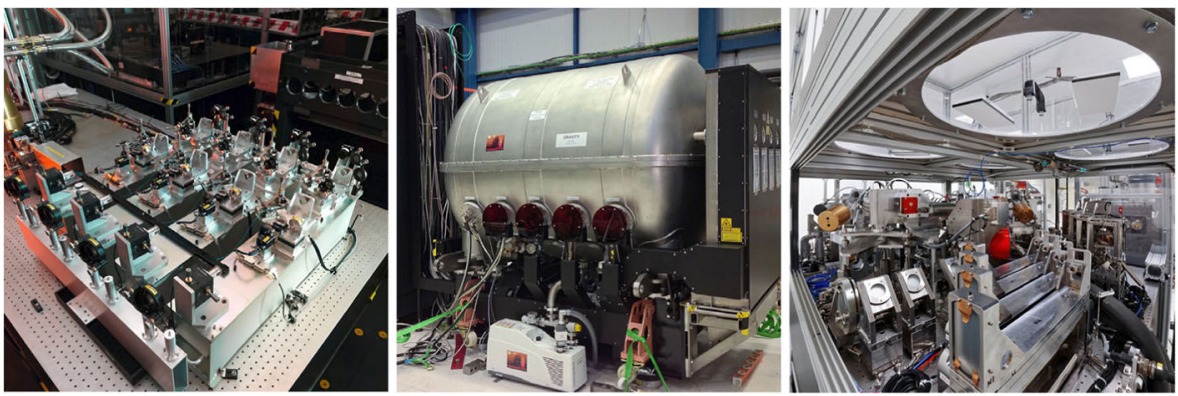

Fig. 2 From 2019 the VLTI interferometric instruments will cover the full atmospheric windows H-band to N-band (1.6-13 $\mu \mathrm{m})$ : left: PIONIER (H-band; in regular operation), center: GRAVITY (K-band; 2016 in commissioning at the VLTI), right: MATISSE (L, M, N-band; photo taken in the lab; VLTI commissioning will commence in early 2018). Image Credits: ESO, B. Lazareff, MPE/GRAVITY team, MATISSE project, Y. Bresson 
hundred targets were accessible with MIDI. Therefore, there is a risk that a GTO target list ${ }^{1}$ could effectively block whole object classes for the non-GTO community, instead of reserving only some prime-targets to the consortia. In these cases, it is an important role of ESO to find a balance between the legitimate interests of the consortia and of the wider community, e.g. by adopting semester-by-semester protection rules and by protecting specific target+instrumentation mode combinations, such that some instrumentation modes are still accessible by non-GTO teams (e.g. continuum versus spectral line observations; astrometric versus visibility modes, etc.).

Balancing interests during instrument commissioning A remarkable development at VLTI has been the development of instruments with modes that have been specifically designed to answer transformational scientific question. Examples are the wideangle astrometric mode of PRIMA (which aimed at the detection of extrasolar planets [3]) and the narrow-angle astrometric mode of GRAVITY (which aims for example at localizing the origin of the flares around Sgr A* and to study strong gravity effects near the supermassive black-hole). Commissioning these technically demanding modes is essential so that the instruments can reach their ultimate science objectives, but it requires also a larger investment of resources and time. These specialised modes push the very frontier of technology, they sometimes reveal deficits in the infrastructure, which, when corrected, are harvested by the whole VLTI user community. On the other hand, there might be standard modes that can be commissioned on short timescales and that might already satisfy the demands of a large fraction of the total user community. Therefore, we consider it the responsibility of ESO together with the instrument consortia to follow a science-wise approach and to find a balance between completing the commissioning of the most advanced instrument modes and in offering standard operational modes.

Potential of large programs Finally, it seems a good time for the community to start developing ideas for large collaborative projects that could help to exploit the opportunities provided by VLTI in a more systematic and comprehensive way than what is possible in standard proposals. Several successful large programs have been conducted with MIDI, namely on AGNs (184.B-0832) and evolved stars (187.D-0924), as well as with PIONIER, where a multiplicity survey on massive stars (189.C-0644) and on Herbig Ae/Be stars (189.C-0963) has been conducted. Such large programs can bring scientists with complementary expertise together. Furthermore, they allow addressing scientific questions that require large object samples, or large time-domain coverage, often creating higher scientific impact than what can be achieved with studies on individual objects, at a given time.

Acknowledgments We acknowledge support from the OPTICON Networking Activity FP7 WP14 and H2020 WP11, funded through the European Commission's 7th Framework R\&D program (grant number 312430) and Horizon 2020 R\&D program (grant number 730890). S.K. acknowledges support from an STFC Rutherford Fellowship (ST/J004030/1).

\footnotetext{
${ }^{1}$ GTO target lists can be accessed using the following website: https:/www.eso.org/sci/observing/telesalloc/gto.html
} 
Open Access This article is distributed under the terms of the Creative Commons Attribution 4.0 International License (http://creativecommons.org/licenses/by/4.0/), which permits unrestricted use, distribution, and reproduction in any medium, provided you give appropriate credit to the original author(s) and the source, provide a link to the Creative Commons license, and indicate if changes were made.

\section{References}

1. Armstrong, J.T., Mozurkewich, D., Rickard, L.J., et al.: ApJ. 496, 550 (1998)

2. Creech-Eakman, M.J., Romero, V., Payne, I., et al.: Proc. SPIE, vol. 9907, p. 990705 (2016)

3. Delplancke, F.: New A.R. 52, 199 (2008)

4. GRAVITY collaboration, et al.: A\&A. 602, 94 (2017)

5. Kervella, P., Coudé du Foresto, V., Glindemann, A., Hofmann, R.: SPIE. 4006, 31K (2000)

6. LeBouquin, J.B., Berger, J.-P., Lazareff, B., et al.: A\&A. 535, A67 (2011)

7. Leinert, C., Graser, U., Przygodda, F., et al.: Ap\&SS. 286, 73 (2003)

8. Matter, A., Lopez, B., Antonelli, P., et al.: Proc. SPIE. 9907, 99070A (2016)

9. Petrov, R.G., Malbet, F., Weigelt, G., et al.: A\&A. 464, 1 (2007)

10. Ten Brummelaar, T.A., McAlister, H.A., Ridgway, S.T., et al.: ApJ. 628, 453 (2005) 